\title{
Robust Cohen-Coon PID Controller for Flexibility of Double Link Manipulator
}

\author{
Ramesh Gamasu ${ }^{1}$ and Venkata Ramesh Babu Jasti ${ }^{2}$ \\ ${ }^{1}$ Research Scholar, Department of EEE, SACET, Cherala (A.P), India \\ ${ }^{2}$ Associate Professor, Department of EEE, RISE Krishna Sai Prakasam Group of \\ Institutions, Ongole (A.P), India \\ ramesh.gamasu@gmail.com ${ }^{1}$,jvrb2010@gmail.com ${ }^{2}$
}

\begin{abstract}
Robots are imitation for the present generation human beings. There is Augechange in between robot and human beings where differentiated by their mind, intellectmal capabilities. Generally Manipulator is an important parameter in robots construction and flexible operation, nothing but robotic arm. In this paper, we proposed a specially designed Controller for the flexible operation of the manipulator is nothing but "Cohen-Coon PID Controller". The proposed PID Controller drives to get flexibilify th operation of double link manipulator. The entire topology has been stwdied, investigated, modeled and simulated on double link manipulator only. The overall simulation for test system can be carried out in control tool box of MAT Lab M-File TeChrique. Each link of double link manipulator was simulated by assigning the state variables in M-File So that highly graphical facilitations are provided for showing the variatron in tip-vibrations and pay-load torque responses for Theta_1 and Theta_2 of dowble link manipulator. However, the Proposed Robust PID Controller shows better performance rather than the normal conventional Closed Loop PID Controllers.

Keywords: Double Link Manipulator, Cohen-Coon PID Controller, Tip-Vibrations, PayLoad Torque Responses, Robust PID Controller
\end{abstract}

\section{Introduction}

The robot technology is advancing rapidly. The industry is moving from the current state of automation to robotization, to increase productivity and to deliver uniform quality. Robots like and robot like manipulators is now commonly employed in hostile environment, such as at various places in an atomic plant for handling radioactive materials. Robots are being employed to construct and repair space stations and satellites. There are now increasing number of applications of robots such as in nursing and aiding a patient .Micro robots are being designed to do damage control inside human veins. Robots like systems are now employed in heavy earth-moving equipment. It is not possible to put up an exhaustive list of robot applications. One type of robot commonly used in the industry is a robotic manipulator or simply a manipulator or a robotic arm [4]. A Manipulator is a machine that has functions similar to human upper limbs, and moves the objects spatially. The Robotics Industries 
Association (RIA) of USA defines the robot as "A reprogrammable, multifunctional manipulator designed to move material through variable programmed motions for the performance of a variety of tasks [3].”

The Review of research work reveals that much work has been done on various aspects of control of manipulators using PID Controllers, nonlinear PD controllers, different algorithms etc., but the results are not generalized fit well to everyone. Thambirajah Ravichandran, David Wang, Glenn Heppler studied on numerical optimization techniques for simultaneously optimizing design parameters of a 2-link rigid manipulator and a nonlinear gain PD controller designed for performing multiple tasks. They cannot make it to study the vector optimization for generating the entire set of optimal solutions to simultaneous design optimization methodology [1].Reza Fotouhi-C, Walerian Szyszkowski, Pete N. Nikiforuk, studied on twd phase trajectory planning for a two link rigid manipulator using two stages. Gepmetric trajectory planning and speed control using a nonlinear scaling of time variable, it was demonstrated that specified velocity is to be followed [2].J.W S. Chong, S.K. Qng, A.Y.C. Nee, K. Youcef-Youmi, studied the use of an augmented reality (AR) environment for facilitating intuitive robot programming, and presents a methodology for planning collision-free paths for an n-d.o.f. (degree-of-freedom) manipulator in a 3D AR environment. The methodology is interactive because the human is involved in defining the free space or collision-free volume (CFV), and selecting the start and goal configurations. António Lopes, Fernand6 Almeida studied on position controlled commercial industrial robot. Combination of the wa manulators behaves as a single manipulator having the impedance and force control dynamic performance of the RCID, as well as the workspace and trajectory tracking bandwidth of the industrial robot. Force-impedance control of the BCID, and experimental results on typical tasks that involve end-effectors contact with uncertain environments of unknown stiffness are presented [5].

\section{Overview of Double LinkManipulator}

To perform an assigned ask or to attain a desired position, a manipulator is required to accelerate from rest, travel at specified path, and finally decelerate to stop. To accomplish this, trajectory, controlling torques is applied by the actuators at the manipulator joints. This torques is computedfrom the equations of motion of the manipulator, which describe the dynamics of the manipulator. The dynamic model is very useful for mechanical design of the structure cholce of actuator, computer simulation of performance, determination of control strategies, and design of control system. The dynamic model and generated trajectory constitute the inputs to the motion-control system of the manipulator [4]. The problem of manipulator control is to find the time behavior of the forces and torques delivered by the actuators for executing the assigned task. Both the manipulator motion control and its force interaction with the environment are monitored by the control algorithm. The above exposed problems will lead to the study of control systems for manipulator and several techniques [2]. 


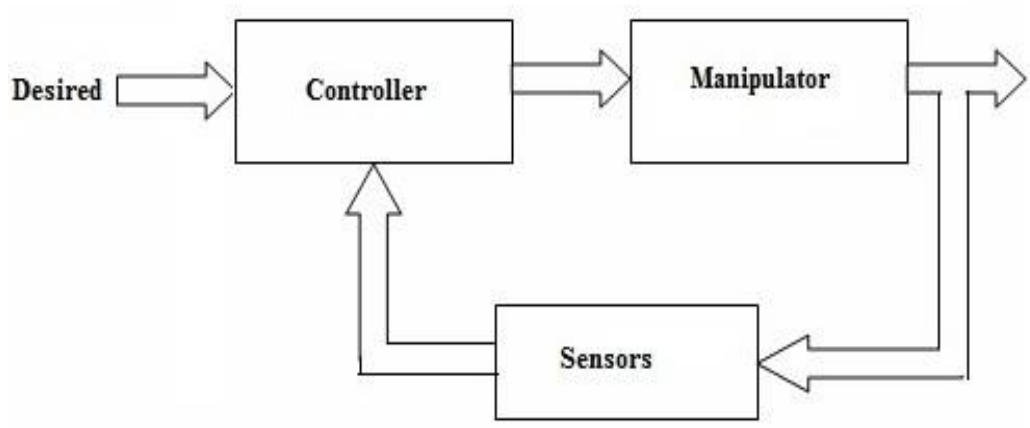

Figure 1. A Schematic Sketch of Manipulator Control System

The tasks to be performed by the manipulator are (1) to move the end-effectors along a desired trajectory, and (2) to extract a force on the environment to carry out the desired task. The controller of manipulator has to control both tasks, the forniter is called position control (or trajectory control) and the latter force control. A schematiasketch of a typical controller is shown below. The positions, velocities, forces, torques are measured by sensors and based on these measurements and the desired behavior, the controller determines the inputs to the actuators on the robot so that the end effectors carriesout the desired task as closely possible [9].

\subsection{Dynamic Modeling}

The dynamics of a simple manipulato is worked out using Lagrange Euler formulation and to sort out the problems invorved in dynamic modeling of 2-link rigid manipulator. Dynamic model of a 2-link rigid manipulator with both rotary joints is obtained using direct geometric approach [7]. For the manlipulator we consider joint variables $\theta 1, \theta_{2}$, link lengths $1_{1}$, $\mathrm{l}_{2}$ and mass of link $\mathrm{m}_{1}$ and $\mathrm{m}_{2}$. Mass of each link is assumed to be a point mass located at the centre of mass of each link. The linear and angular velocities are $\mathrm{v}_{1}, \mathrm{v}_{2}$ and $\theta_{1}$ and $\theta_{2}$ respectively [6].

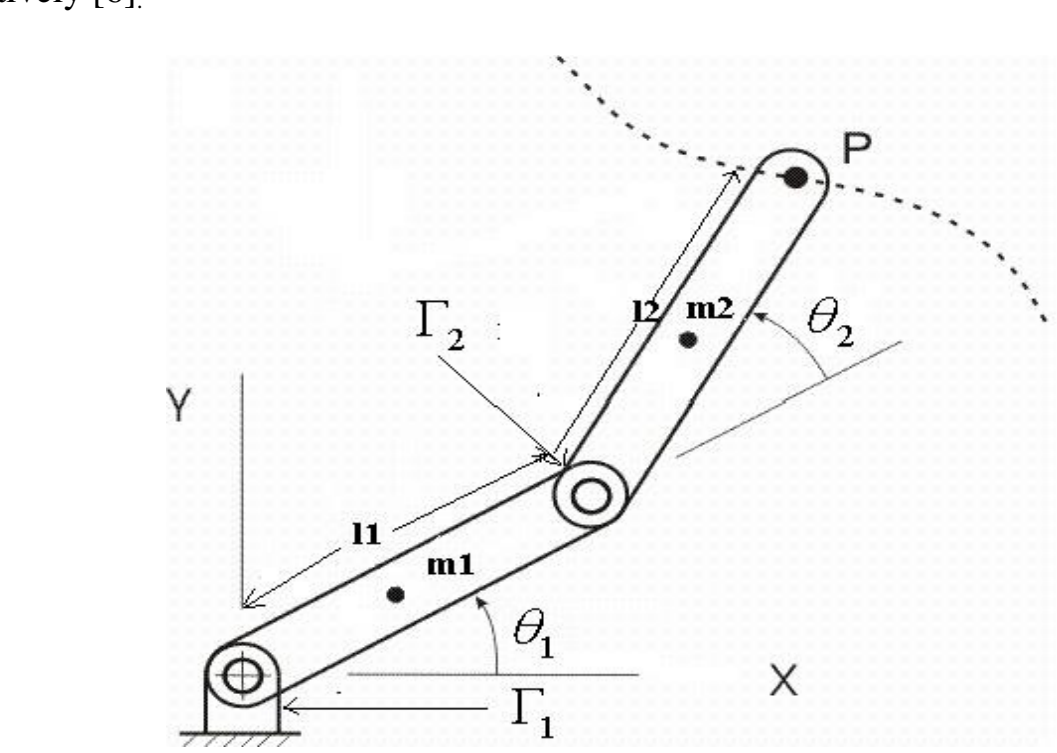

Figure 2. Model of two link rigid manipulator 
The Lagrangian requires kinetics and potential energies of the manipulator. The kinetic energy of a rigid body (a link), can be expressed as

$\mathrm{K}=\frac{1}{2} \mathrm{~m} v^{2}+\frac{1}{2} \mathrm{I} \omega^{2}$

Where $v$ is the linear velocity, $\omega$ is the angular velocity, $m$ is the mass, and $I$ is the moment of inertia of the rigid body at its center of mass .

Thus, the kinetic energy for the link 1 with liner velocity $v_{1}=\frac{1}{2} L_{1} \dot{\theta}_{1}$, angular velocity $\omega_{1}=\dot{\theta}_{1}$, moment of inertia $I_{1}=\frac{1}{12} m_{1} L_{1}^{2}$, and mass $m_{1}$ is

$K_{1}=\frac{1}{2} m_{1} v_{1}^{2}+\frac{1}{2} I_{1} \omega_{1}^{2}=\frac{1}{8} m_{1} L_{1}^{2} \dot{\theta}_{1}^{2}+\frac{1}{24} m_{1} L_{1}^{2} \dot{\theta}_{1}^{2}={ }_{6}^{1} m_{1} L_{1}^{2} \dot{\theta}_{1}^{2}$

And its potential energy is

$P_{1}=\frac{1}{2} m_{1} g L_{1} \sin \theta_{1}$

Because both the joints are revolute, the generalized torques $\tau_{1}$ and $\tau_{2}$ represent the actual joint torques.

$$
\begin{aligned}
& \tau_{1}=M_{11} \theta_{1}^{*}+M_{21} \theta_{2}^{*}+H_{1}+G_{1} \\
& \mathrm{\tau}_{2}=M_{21} \theta_{1}^{*}+M_{22} \theta_{2}^{*}+H_{2}+G_{2} \\
& {\left[\begin{array}{l}
\Gamma_{1} \\
\Gamma_{2}
\end{array}\right]=\left[\begin{array}{ll}
M_{11} & M_{12} \\
M_{21} & M_{22}
\end{array}\right]\left[\begin{array}{l}
\ddot{\theta}_{1} \\
\ddot{\theta}_{2}
\end{array}\right]+\left[\begin{array}{cc}
-2 H \dot{\theta}_{2} & -H \dot{\theta}_{2} \\
H \dot{\theta}_{1} & \mathbb{Q}
\end{array}\right]\left[\begin{array}{l}
\dot{\theta}_{1} \\
\dot{\theta}_{2}
\end{array}\right]+\left[\begin{array}{l}
G_{1} \\
G_{2}
\end{array}\right]} \\
& M_{11}=\left[\left(\frac{1}{3} m_{1}+m p_{2}\right)+\left(\frac{1}{3} m_{2}\right) L_{2}+m_{2} L_{1} L_{2} C_{2}\right] \\
& M_{21}=M_{12}=m_{2}\left[\frac{1}{3} L_{2}^{2}+\frac{1}{2} L_{1}\left(C_{2}\right]\right. \\
& M_{22}=\frac{1}{3} m_{2} L_{2}^{2} \\
& H_{1}=-m_{2} L_{1} L_{2} S_{2} \mathcal{Y}\left(\theta_{1}^{\prime} \theta_{2}^{\prime}\right)-\frac{1}{2} m_{2} L_{1} L_{2} S_{2} \theta_{2}^{2} \\
& H_{2}=m_{2} L_{1} L_{2} S_{2} \dot{\theta}_{1}^{2} \\
& G_{1}=\left(\frac{1}{2} m_{1}+m_{2}\right) g L_{1} C_{1}+\frac{1}{2} m_{2} g L_{2} C_{12} \\
& G_{2}=\frac{1}{2} m_{2} g L_{2} C_{12}
\end{aligned}
$$

These coefficients are defined as

$M_{i \bar{i}}=$ effective inertia

$M_{i j}=$ Effective coupling inertia, 
$H_{i}=$ centrifugal and carioles acceleration forces

This direct formation approach becomes quite cumbersome when a manipulator with more than 2-DOF is analyzed, in the following sections, the derivation of EOM for an $n$ - DOF manipulator, based on homogeneous coordinate transformation matrices is presented [9].

\section{Proposed Cohen-Coon PID Controller}

A proportional-integral-derivative controller (PID controller) is a generic control loop feedback mechanism widely used in industrial control systems. A Cohen-Coon PID Controller attempts to correct the error between a measured process variable and a desired set point by calculating and then outputting a corrective action that can adjust the process accordingly. The Cohen-Coon PID Controller calculation (algorithm) involves three separate parameters; the Proportional, the Integral and Derivative values. The Proportional value determines the reaction to the current error, the Integral determines the reaction based on the sum of recent errors and the Derivative determines the reaction to the rate at which the error has been changing [11].

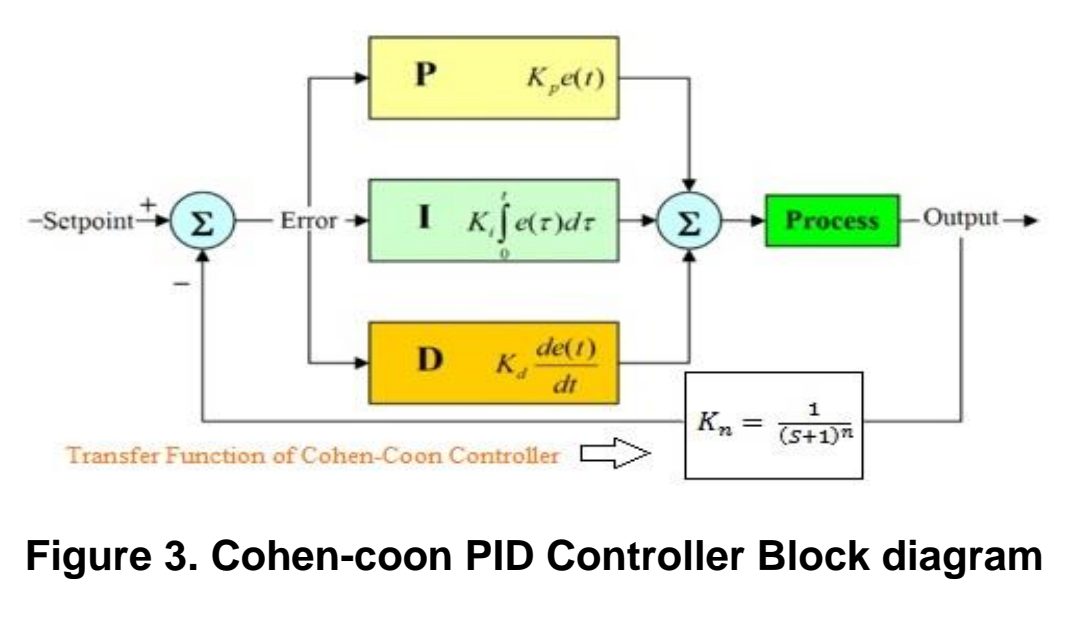

The weighted sum of these three actions is used to adjust the process via a control element such as the position of a controlvalve or the power supply of a heating element.

By "tuning" the three constants in the Cohen-Coon PID Controller algorithm the PID can provide control action designed for specific process requirements. The response of the controller can be described in terms of the responsiveness of the controller to an error, the degree to which the controller overshoots the set point and the degree of system oscillation. Note that the use of the PID algorithm for control does not guarantee optimal control of the system or system stability. Some applications may require using only one or two modes to provide the appropriate system control. This is achieved by setting the gain of undesired control outputs to zero [4]. A Cohen-Coon PID Controller will be called a PI, PD, P or I controller in the absence of the respective control actions. PI controllers are particularly common, since derivative action is very sensitive to measurement noise, and the absence of an integral value may prevent the system from reaching its target value due to the control action [10]. 


\subsection{Control Strategy}

If the PID controller parameters (the gains of the proportional, integral and derivative terms) are chosen incorrectly, the controlled process input can be unstable, i.e., its output diverges, with or without oscillation, and is limited only by saturation or mechanical breakage. Tuning a control loop is the adjustment of its control parameters (gain/proportional band, integral gain/reset, derivative gain/rate) to the optimum values for the desired control response. The optimum behavior on a process change or set point change varies depending on the application. Some processes must not allow an overshoot of the process variable beyond the set point if, for example, this would be unsafe. Other processes must minimize the energy expended in reaching a new set point $[6,3]$.

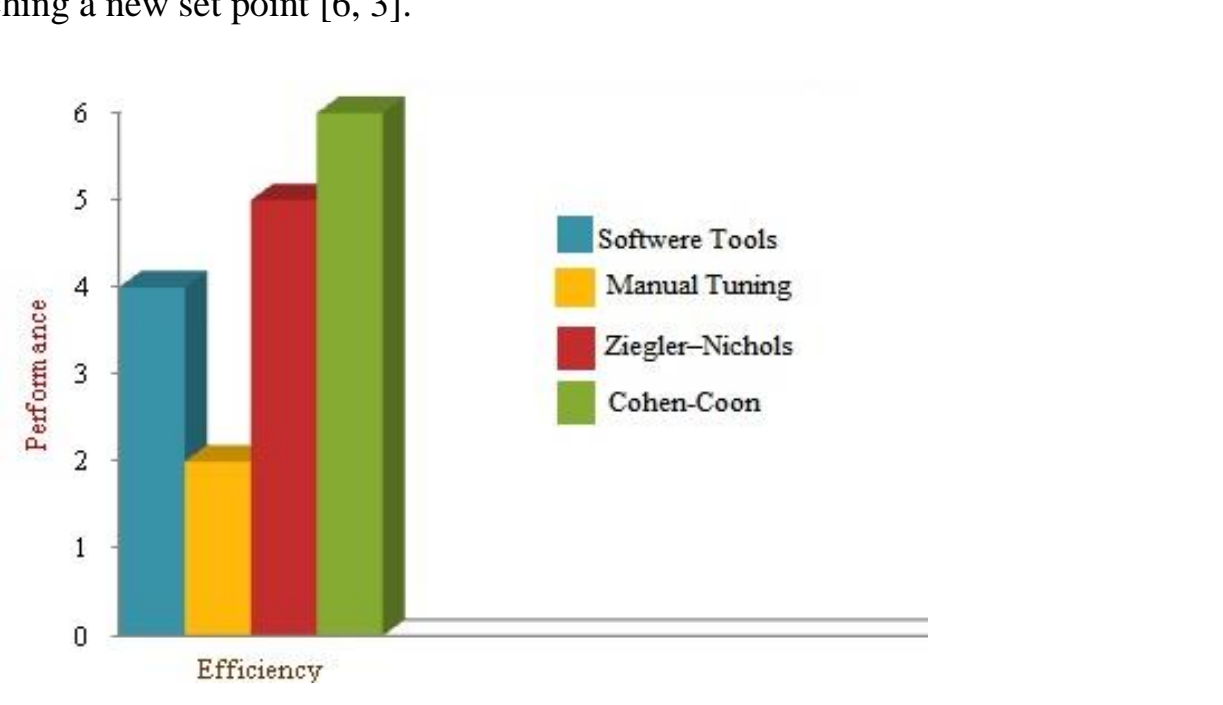

Figure 4. Comparison of Proposed Controller with Other Controllers

Generally, stability of response (theneverse of instability) is required and the process must not oscillate for any combination of process conditions and set points. Some processes have a degree of non-linearity and so parameters that work well at full-load conditions don't work when the process is starting gp from no-load. This section describes some traditional manual methods for loop tuning There are several methods for tuning a PID loop. The most effective methods generally involye the development of some form of process model, and then choosing P, I, and D based on the dynamic model parameters. Manual tuning methods can be relatively inefficient. The choice of method will depend largely on whether or not the loop can be taken "offline" for tuning, and the response time of the system. If the system can be taken offline, the best tuning method often involves subjecting the system to a step change in input, measuring the output as a function of time, and using this response to determine the control parameters [7]. 


\section{Modeling and Simulation of Double Link Manipulator with Proposed Controller}

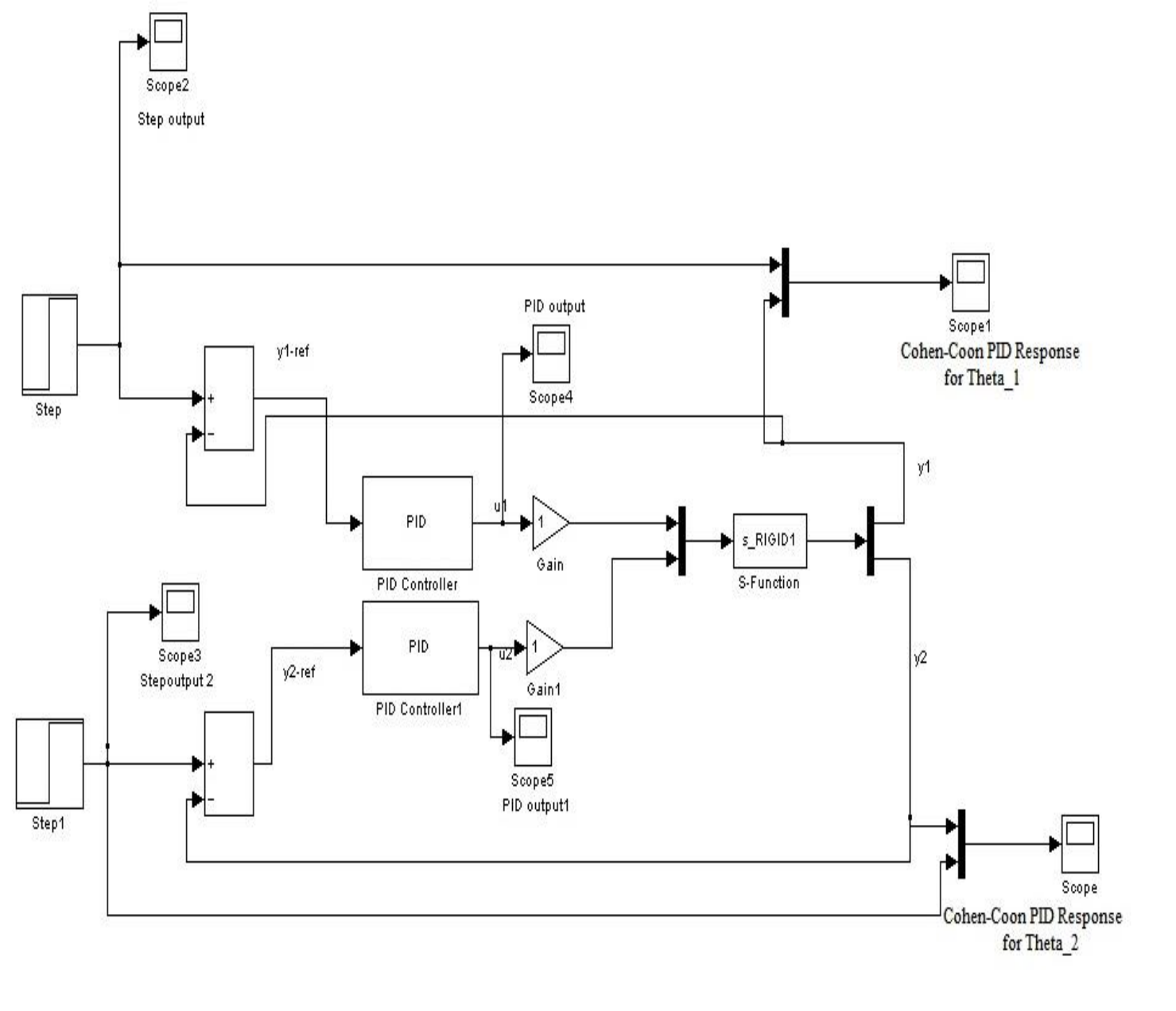

Figure 5. Simulation of Double link Manipulator using Cohen-Coon PID Controller using State Space Technique

\section{Results and Discursions}

The test System of double link manipulator was modeled and simulated in MAT Lab/Simulink Control tool box. Here the electro mechanical model was initiated by using state space representation and the each aspect of proposed test system assigned as a state variables. The simulation results are evaluated for Theta_1 and Theta_2 are the first and second links of double link manipulator. Here, the two arms of two link manipulator named as a first link arm and second link arm. Theta_1 and Theta_2 are the two responses for two link manipulator. 


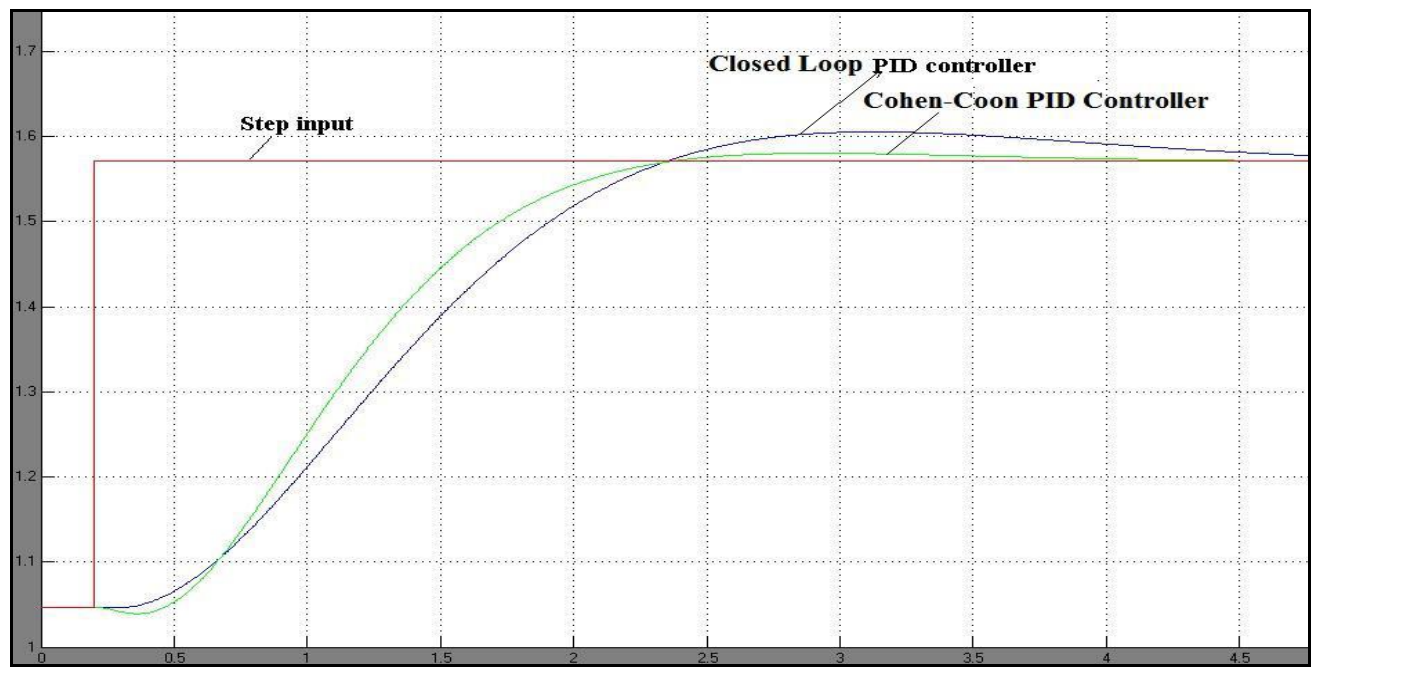

Figure 6. Pay-Load Torque Response for Theta 1

Figure 6 Shows Compared Responses for Pay-Load Torque Responses lor Theta_1 from Closed PID Response to Cohen-Coon PID Controller response respectively. The proposed graphical representation initiates initial Theta_1 response of one of the links of double link manipulator. Here, Theta_1 represents response caused by first Tink of manipulator. This Theta_1 consisting a combination of pay load torque responses and tip vibrations. In the Figure 6, on $\mathrm{x}$-axis time and $\mathrm{y}$-axis pay 10 ad torque was represented. From the graphical observations the open loop pay-load torque is somewlat less and in case of closed loop it is increases to $4-5 \%$ in case of Cohen-coon proposed controller.

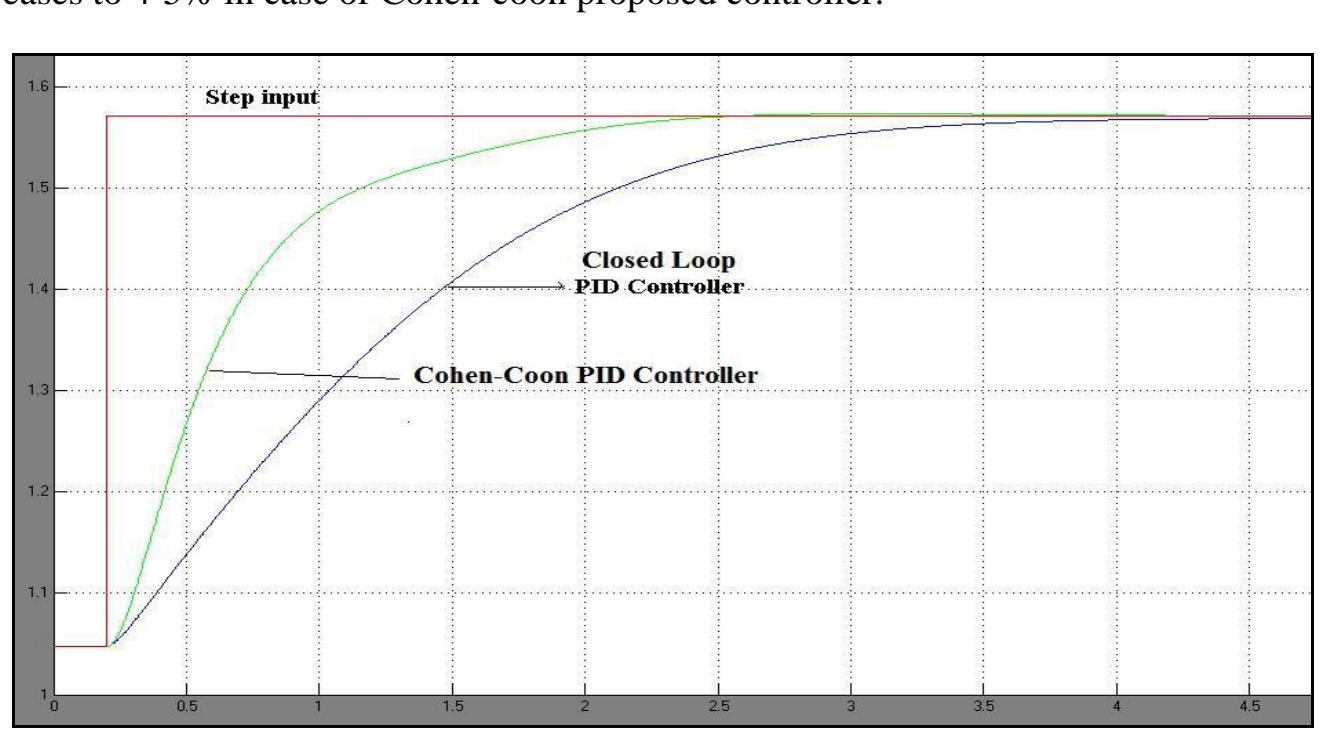

Figure 7. Pay-Load Torque Response for Theta_2

Figure 7 shows the Pay-Load Torque Response for Theta_2 from Closed Loop PID Controller to Cohen-Coon PID Controller respectively. The evaluated response was a response for Theta_2.Here the comparison between closed loop and Cohen-coon PID Controller has represented. The compared responses initiate that improved change over occurring from Closed PID to Cohen-Coon PID. The pay-load response for closed loop 
controller is much less with respect to Cohen-Coon PID Controller. Practically, through comparison the response caused by open loop controller is less with respect to closed loop and Cohen-Coon Controller is more through comparison of Closed loop PID. In the proposed graphical representation, on $\mathrm{x}$-axis time and $\mathrm{y}$-axis pay load torque response has represented

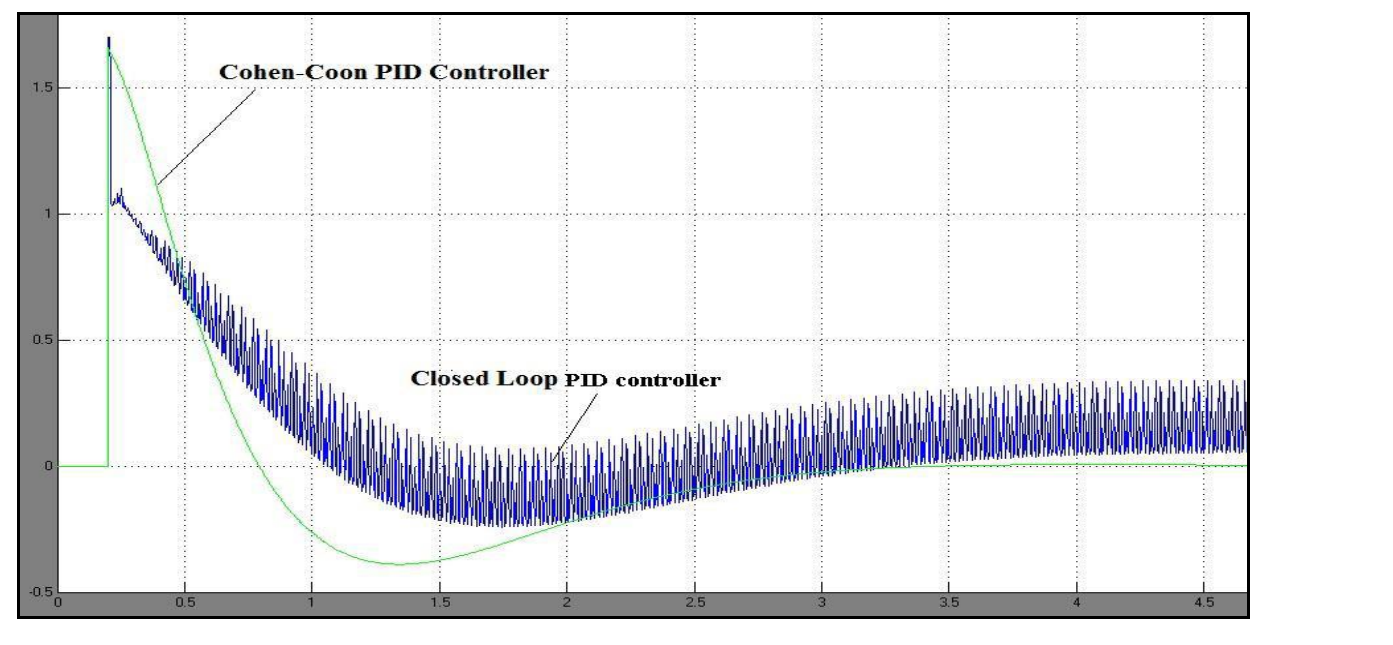

Figure 8. Tip-Vibrations Response for Theta_ 1

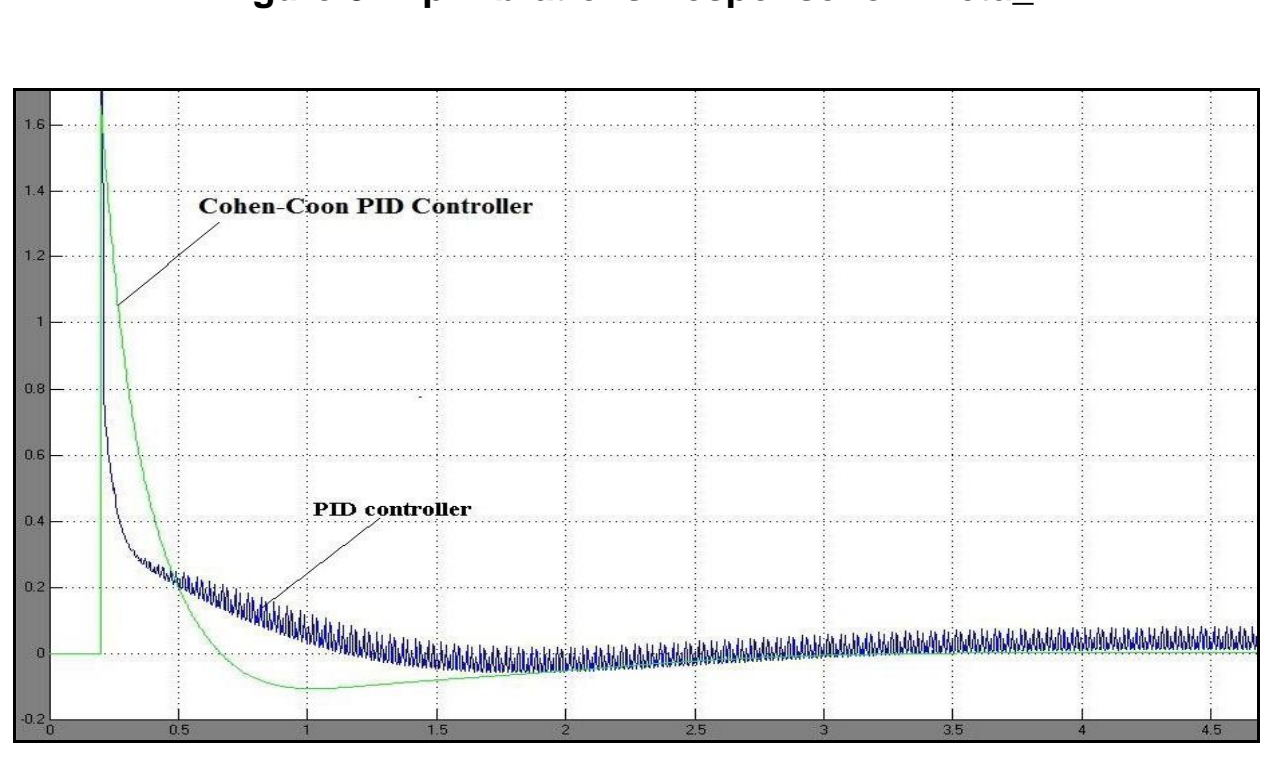

Figure 9. Tip-Vibrations Response for Theta_2

Figure 8 represents tip -vibrations for Theta_1 of Double link manipulator. These both responses are obtained by using state variable representation. The proposed graphical representation initiates the difference shown between tip-vibrations of conventional closed loop PID Controller to Cohen-coon PID Controller. The tip vibrations are shows more effect, in case of a conventional closed controller in active condition and the same effects less when it is executed with proposed PID Controller. The maximum disturbance occurring due to closed loop is very much greater than that of the robust PID Controller, i.e., Cohen-Coon PID Controller. The passage of decaying disturbance from Conventional closed loop to Cohencoon is $30-40 \%$. 
Figure 9 is also represents the same tip-vibrations of Theta_2 of one ram of the double link manipulator. Here, on $\mathrm{x}$-axis time and $\mathrm{y}$-axis tip-vibrations are represented. The proposed graphical representation shows the variation between closed loop conventional PID Controller responses to Robust Cohen-coon controller. From the figure, the response caused by conventional PID Controller rends some disturbances at far end of double link manipulator.

The respective noise or disturbance was eliminated by involving the back to back controller with " $n$ " number of iterations leads to a more flexibility in the manipulator.

The proposed PID Controller acts as a back to back controller, initiates robustness in the manipulator each arm. The overall duo responses of Theta_1 and Theta_2 for tip -vibrations are more effective for the proposed robust Cohen-coon controller.

\section{Conclusions}

The overall double link manipulator test system was studied, modeled and simulated. Flexibility is an important aspect in the view of manipulators. Flexibility is possible only when using a proper control methodology. Proposed Control méthodology is achieved by utilizing a robust Cohen-coon PID Controller. This control responds for grater rise time, and maximum peak over shoot, less time period to take off for settled the response. Anyway, the proposed controller acts as back-back controller for flexibility in double link manipulator.

In this article, the case study continued on comparison between/a closed loop PID Controller and Cohen-Coon PID Controller. The Overall topology explains the pay-load torque and tip-vibrations of manipulator due to closed loop controller causes $50 \%$ flexibility in each arm and the same parameters are due to Cohen-Coon controller causes maximum 60$70 \%$ of flexibility. Hence the proposed Cohen-coon PID Controller acts as a robust controller in the aspect of better performance charectestics.

\section{Acknowledgements}

Authors are expressing their sincene thanks to all the faculty of Electrical \& Electronics Engineering Department and the management of RISE Krishna Sai Prakasam group of institutions, Ongole.

\section{References}

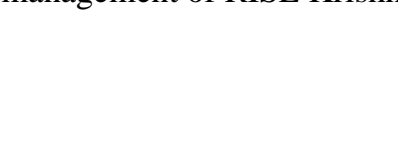

[1] T. Ravichandran, D. Wang and G. Hieppler, "Simultaneous plant-controller design optimization of a two-link planar manipulator", Journal 6f Mechatronics, vol. 16, (2006), pp. 233-242.

[2] R. Fotouhi-C, W. Szyszkowski and P. N. Nikiforuk, "Trajectory planning speed control for a two-link rigid manipulator", Journal of mechanical design, vol. 124, (2002), pp. 585-589.

[3] R. Fotouhi-C, PANikiforuk and W. Szyszkowski, "Combined trajectory planning and parameter identification of a two link rigid manipulator", Proceedings of ASME Design Engineering Technical conferences, Atlanta, Georgia, (1998.

[4] R. Fotouhi-C, W. Szyszkowski, P. N. Nikiforuk and M. M. Gupta, "Parameter identification and trajectory folloxing of a two link rigid manipulator", Journal of Systems and Control Engineering, proceedings part I, (2000), $\mathrm{pp} / 213$.

[5] J. W. S. Chong, S. K. Ong, A. Y. C. Nee and K. Youcef-Youmi, "Robot programming using augmented reality: An interactive method for planning collision free paths", Journal of Robotics and Computer Integrated manufacturing, (2004).

[6] A. Lopes and F. Almeida, "A force impedance controlled industrial robot using an active robotic auxiliary device", Journal of Robotics and Computer - Integrated manufacturing, vol. 24, (2008), pp. 299309.

[7] K. Abdel-Malek and J. Yang, "Sweeping of an object held by a robotic end-effector", Journal of Robotics and Computer - Integrated manufacturing, vol. 21, (2005), pp. 159-173.

[8] D. Oetomo and M. H. Ang Jr, "Singularity robust algorithm in serial manipulators", Journal of Robotics and Computer - Integrated manufacturing, (2007). 
[9] A. Gasparetto and V. Zanotto, "A technique for time-jerk optimal planning of robot trajectories", Journal of Robotics and Computer - Integrated manufacturing, vol. 24, (2008), pp. 415-426.

[10] K. A. Atia and M. P. Cartmell, "A general dynamic model for a large scale 2-DOF Planar parallel manipulator", Robotica, vol. 17, no. 6, (1999), pp. 675-684.

[11] Z. Shiller and H. H. Lu, "Computation of path constrained time optimal control motions with dynamic singularities", Transaction of ASME Journal of Dynamic Systems, Measurement and Control, vol. 114, no. 2, (1998), pp. 34-40.

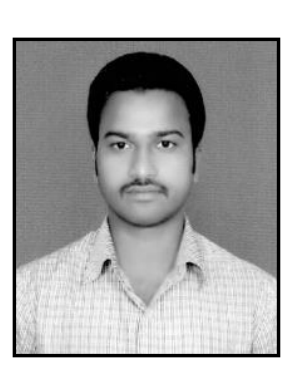

\section{Authors}

Mr. Ramesh Gamasu was born in India, on October 15, 1989. He obtained his B.Tech Degree in Electrical and Electronics Engineering from JNT University, Kakinada (A.P), India in 2010.He worked as assistant professor of Electrical Science Engineering Department in ${ }^{\bullet}$ Centurion University of Technology \& Management, Perlakhemundi (Odisha) and Electrical Engineering Department of RISE Krishna Sai Group of Institutions, Ongole (A.P). Currently, working as Research Scholar in Department of Electrical \& Electrontcs Engineering, St.Ann's College of Engineering \& Technology Cherala (A.P) - India. He is member of various Engineering societies like IAENG, IACSIT, IACSE, ASEE, and UACEE, IAEM etc. He published various research articles and letters on Power Systems Engineering. His areas of interest include Power Systems Deregulation and reconstruction, role of artificial techniques for diagnosing the power quality problems and Power Systems Dynamics, etc

Mr. Venkata Ramesh Babu Jasti was born in Chimakurthy, Andhra

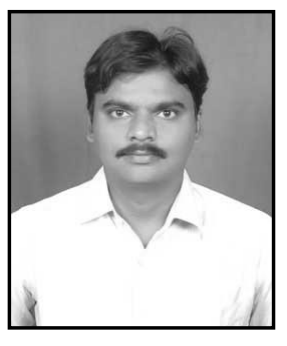
Pradesh, on July 11, 1982. He 19 ceived his B.Tech Degree in Electrical and Electronics Engineering from JNT University, Hyderabad (A.P), India in 2004 and M.E. degree in Power Electronics \& Industrial Drives from Sathyabhama University Chennai (T.N) in 2008. Currently, he is Associate Professor and Head of the Department of Electrical \&Electronics Engineering Department in RISE Krishna Sai Prakasam Group of Institutions, Ongole (A.P). He is a life member of ISTE. He published various research articles and letters on Power Systems Engineering. His areas of interest include Power Systems Deregulation and reconstruction, role of artificial techniques for diagnosing the power quality problems and Power Systems Dynamics, etc.

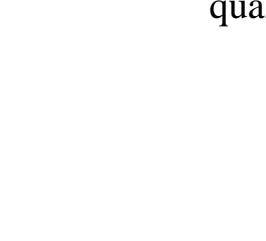


International Journal of Control and Automation

Vol.7, No.1 (2014)

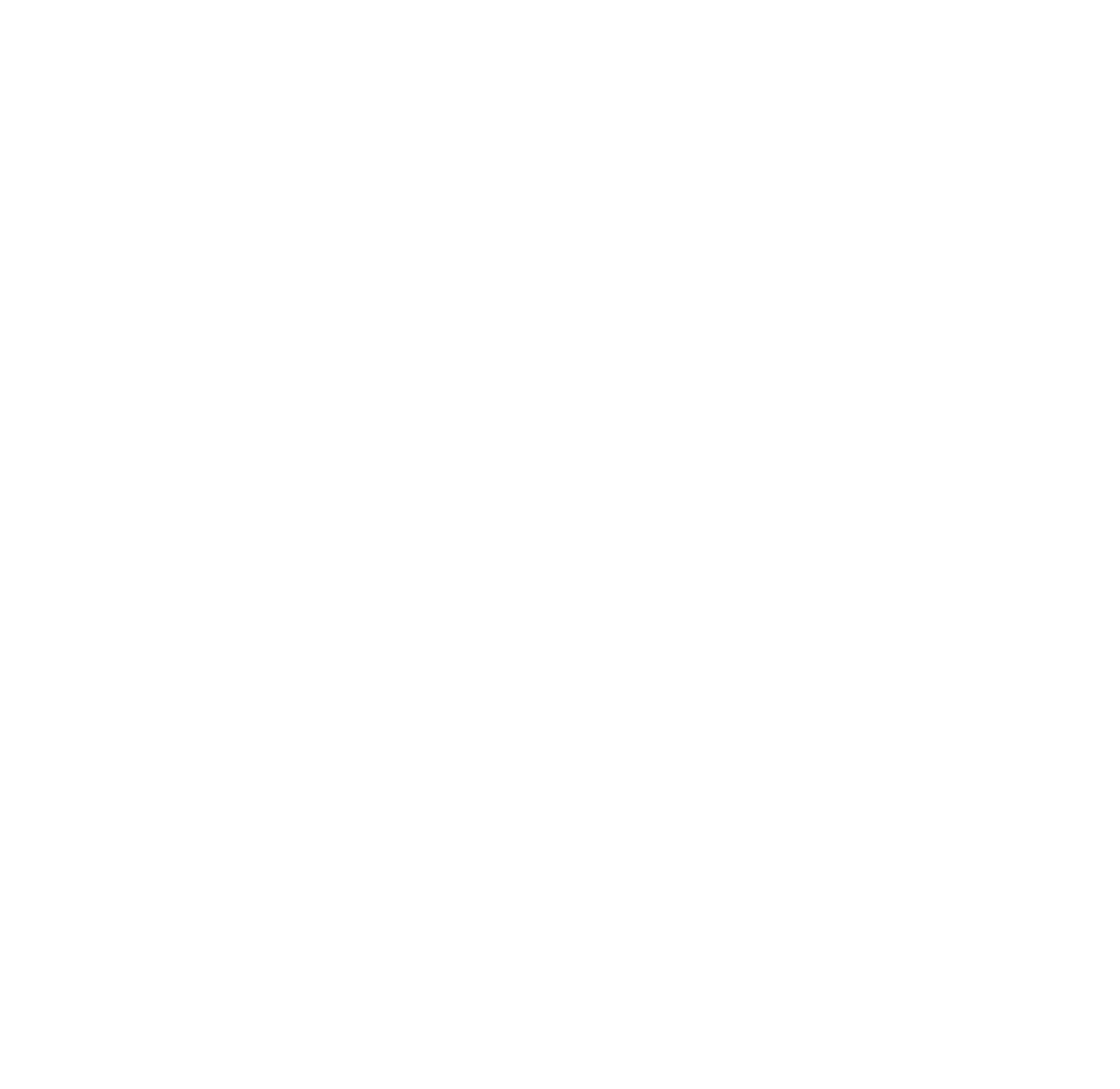

